\title{
Front Matter: Volume 8031
}

, "Front Matter: Volume 8031," Proc. SPIE 8031, Micro- and Nanotechnology Sensors, Systems, and Applications III, 803101 (23 May 2011); doi: 10.1117/12.900910

SPIE Event: SPIE Defense, Security, and Sensing, 2011, Orlando, Florida, United SPIE. States 


\title{
PROCEEDINGS OF SPIE
}

\section{Micro- and Nanotechnology Sensors, Systems, and Applications III}

\author{
Thomas George \\ M. Saif Islam \\ Achyut K. Dutta \\ Editors
}

25-29 April 2011

Orlando, Florida, United States

Sponsored and Published by

SPIE

Volume 8031

Part One of Two Parts 
The papers included in this volume were part of the technical conference cited on the cover and title page. Papers were selected and subject to review by the editors and conference program committee. Some conference presentations may not be available for publication. The papers published in these proceedings reflect the work and thoughts of the authors and are published herein as submitted. The publisher is not responsible for the validity of the information or for any outcomes resulting from reliance thereon.

Please use the following format to cite material from this book:

Author(s), "Title of Paper," in Micro- and Nanotechnology Sensors, Systems, and Applications III, edited by Thomas George, M. Saif Islam, Achyut K. Dutta, Proceedings of SPIE Vol. 8031 (SPIE, Bellingham, WA, 2011) Article CID Number.

ISSN 0277-786X

ISBN 9780819486059

Published by

SPIE

P.O. Box 10, Bellingham, Washington 98227-0010 USA

Telephone +1 3606763290 (Pacific Time) · Fax +1 3606471445

SPIE.org

Copyright (C) 2011, Society of Photo-Optical Instrumentation Engineers

Copying of material in this book for internal or personal use, or for the internal or personal use of specific clients, beyond the fair use provisions granted by the U.S. Copyright Law is authorized by SPIE subject to payment of copying fees. The Transactional Reporting Service base fee for this volume is $\$ 18.00$ per article (or portion thereof), which should be paid directly to the Copyright Clearance Center (CCC), 222 Rosewood Drive, Danvers, MA 01923. Payment may also be made electronically through CCC Online at copyright.com. Other copying for republication, resale, advertising or promotion, or any form of systematic or multiple reproduction of any material in this book is prohibited except with permission in writing from the publisher. The CCC fee code is 0277-786X/11/ \$18.00.

Printed in the United States of America.

Publication of record for individual papers is online in the SPIE Digital Library.

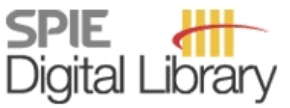

SPIEDigitalLibrary.org

Paper Numbering: Proceedings of SPIE follow an e-First publication model, with papers published first online and then in print and on CD-ROM. Papers are published as they are submitted and meet publication criteria. A unique, consistent, permanent citation identifier (CID) number is assigned to each article at the time of the first publication. Utilization of CIDs allows articles to be fully citable as soon as they are published online, and connects the same identifier to all online, print, and electronic versions of the publication. SPIE uses a six-digit CID article numbering system in which:

- The first four digits correspond to the SPIE volume number.

- The last two digits indicate publication order within the volume using a Base 36 numbering system employing both numerals and letters. These two-number sets start with $00,01,02,03,04$, $05,06,07,08,09,0 A, 0 B \ldots 0 Z$, followed by 10-1Z, 20-2Z, etc.

The CID number appears on each page of the manuscript. The complete citation is used on the first page, and an abbreviated version on subsequent pages. Numbers in the index correspond to the last two digits of the six-digit CID number. 


\title{
Contents
}

\section{Part One}

\author{
xiii Conference Committee \\ xvii Introduction
}

SESSION 1 DIP PEN NANOLITHOGRAPHY

803102 Tip-based nanofabrication: an approach to true nanotechnology (Keynote Paper) [8031-01]

K. P. Bloschock, A. R. Schofield, System Planning Corp. (United States); T. W. Kenny, Stanford Univ. (United States)

803103 Direct-write scanning probe lithography: towards a desktop fab (Invited Paper) [8031-02]

L. R. Giam, A. J. Senesi, X. Liao, L. S. Wong, J. Chai, D. J. Eichelsdoerfer, W. Shim, B. Rasin,

S. He, C. A. Mirkin, Northwestern Univ. (United States)

803104 Tip-based patterning of HOPG and CVD graphene (Invited Paper) [8031-03]

B. T. Hicks, N. Yoshimizu, Cornell Univ. (United States); C. O' Connell, Univ. of Rhode Island (United States); A. Lal, C. R. Pollock, Cornell Univ. (United States)

803105 Nanofabrication using heated probe tips (Invited Paper) [8031-04]

J. R. Felts, P. C. Fletcher, S. Somnath, J. Pikul, Univ. of Illinois at Urbana-Champaign (United States); Z. Dai, Advanced Diamond Technologies, Inc. (United States); W. K. Lee,

P. E. Sheehan, U.S. Naval Research Lab. (United States); W. P. King, Univ. of Illinois at Urbana-Champaign (United States)

803106 Laser-assisted nanoprocessing and growth of semiconductor nanostructures (Invited Paper) [8031-05]

D. J. Hwang, S.-G. Ryu, E. Kim, J.-H. Yoo, Univ. of California, Berkeley (United States); B. Xiang, O. Dubon, A. M. Minor, Univ. of California, Berkeley (United States) and Lawrence Berkeley National Lab. (United States); C. P. Grigoropoulos, Univ. of California, Berkeley (United States)

803107 Semiconductor nanomembranes: a platform for new science and technology (Keynote Paper) [8031-06]

M. G. Lagally, A. M. Kiefer, D. M. Paskiewicz, F. Cavallo, S. A. Scott, Z. Q. Ma, D. E. Savage, Univ. of Wisconsin-Madison (United States)

803109 Si pillar structured thermal neutron detectors: fabrication challenges and performance expectations (Invited Paper) [8031-08]

R. J. Nikolic, Q. Shao, L. F. Voss, A. M. Conway, R. Radev, T. F. Wang, Lawrence Livermore National Lab. (United States); M. Dar, N. Deo, C. L. Cheung, Univ. of Nebraska-Lincoln (United States); L. Fabris, C. L. Britton, M. N. Ericson, Oak Ridge National Lab. (United States) 
8031 OA The role of THz and submillimeter wave technology in DHS (Keynote Paper) [8031-09] T. Coty, U.S. Dept. of Homeland Security (United States); A. Fuller-Tedeschi, Strategic Analysis, Inc. (United States)

8031 OB High-performance heterostructure backward diode detectors (Invited Paper) [8031-10] P. Fay, Z. Zhang, Univ. of Notre Dame (United States)

8031 OC A micro-fabricated sheet-beam Orotron THz source (Invited Paper) [8031-11] S. J. Papadakis, J. A. Hoffmann, A. H. Monica, D. Deglau, The Johns Hopkins Univ. Applied Physics Lab. (United States); J. Yu, T. Antonsen, G. Nusinovich, Univ. of Maryland, College Park (United States); R. Osiander, The Johns Hopkins Univ. Applied Physics Lab. (United States)

8031 OD MEMS-based uncooled THz detectors for staring imagers (Invited Paper) [8031-12] J. A. Cox, R. Higashi, F. Nusseibeh, C. Zins, Honeywell ACS Sensor \& Wireless Lab. (United States)

8031 OE Integrated chip-scale THz technology (Invited Paper) [8031-13]

M. C. Wanke, Sandia National Labs. (United States); M. Lee, Sandia National Labs. (United States) and The Univ. of Texas at Dallas (United States); C. D. Nordquist, M. J. Cich, M. Cavaliere, A. M. Rowen, J. R. Gillen, C. L. Arrington, Sandia National Labs. (United States); A. D. Grine, LMATA Govt. Services (United States); C. T. Fuller, J. L. Reno, Sandia National Labs. (United States)

\section{SESSION 4 THZ CHARACTERIZATION OF SEMICONDUCTOR MATERIALS}

8031 OF Programmatic perspectives with technical examples for THz materials characterization (Keynote Paper) [8031-14]

W. Buchwald, Air Force Research Lab. (United States)

$80310 \mathrm{THz}$ characterization of hydrated and anhydrous materials (Invited Paper) [8031-15] A. Sokolnikov, Visual Solutions \& Applications (United States)

$8031 \mathrm{OH} \quad$ 2DEG GaN hot electron microbolometers and quantum cascade lasers for THz heterodyne sensing (Invited Paper) [8031-16]

R. Ramaswamy, K. Wang, A. Stier, Univ. at Buffalo, SUNY (United States); A. Muraviev, Univ. at Buffalo, SUNY (United States) and Rensselaer Polytechnic Institute (United States); G. Strasser, A. Markelz, Univ. at Buffalo, SUNY (United States); M. Shur, Rensselaer Polytechnic Institute (United States); R. Gaska, Sensor Electronic Technology, Inc. (United States); A. Sergeev, V. Mitin, Univ. at Buffalo, SUNY (United States)

8031 OJ Silicon and nitride FETs for THz sensing (Invited Paper) [8031-18]

M. Shur, Rensselaer Polytechnic Institute (United States)

8031 OK Terahertz QC-lasers and active metamaterial antennas (Invited Paper) [8031-19]

B. S. Williams, P. Hon, A. Tavallaee, T. Itoh, Univ. of California, Los Angeles (United States);

Q. Chen, Northrop Grumman Aerospace Systems (United States) 
8031 OM MEMs adaptive optics at the Naval Research Laboratory (Invited Paper) [8031-21] S. R. Restaino, J. R. Andrews, T. Martinez, C. C. Wilcox, F. Santiago, U.S. Naval Research Lab. (United States); D. M. Payne, Narrascape (United States)

8031 ON Actuation for deformable thin-shelled composite mirrors (Invited Paper) [8031-22] C. C. Wilcox, U.S. Naval Research Lab. (United States); D. V. Wick, G. Soehnel, B. E. Bagwell, Sandia National Labs. (United States); R. C. Romeo, R. N. Martin, Composite Mirror Applications, Inc. (United States); M. S. Baker, N. L. Breivik, B. L. Boyce, Sandia National Labs. (United States); T. Martinez, S. R. Restaino, U.S. Naval Research Lab. (United States)

$803100 \quad$ Micro ion frequency standard (Invited Paper) [8031-23]

P. D. D. Schwindt, Y.-Y. Jau, H. Partner, D. K. Serkland, R. Boye, L. Fang, A. Casias, R. P. Manginell, M. Moorman, Sandia National Labs. (United States); J. Prestage, N. Yu, Jet Propulsion Lab. (United States)

8031 OP Imaging a linearly or circularly polarized scene: micro-components and shrimp (Invited Paper) [8031-24]

S. A. Kemme, D. A. Scrymgeour, A. R. Ellis, A. A. Cruz-Cabrera, R. R. Boye, J. R. Wendt,

T. R. Carter, S. Samora, Sandia National Labs. (United States)

\section{SESSION 6 NANOPHOTONICS}

8031 OR Optical components for lab-in-a-tube systems (Invited Paper) [8031-26]

E. J. Smith, Institute for Solid State and Materials Research (Germany); Y. Mei, Institute for Solid State and Materials Research (Germany) and Fudan Univ. (China); O. G. Schmidt, Institute for Solid State and Materials Research (Germany)

8031 OS Integrated microsystems for molecular pathology (Invited Paper) [8031-27]

A. Scherer, G. Maltezos, I. Malik, E. Kartalov, California Institute of Technology (United States)

\section{SESSION 7 PHOTON TRAPPING WITH ID STRUCTURES AND NOVEL DEVICE APPLICATIONS}

8031 OT Efficient light-trapping nanostructures in thin silicon solar cells (Invited Paper) [8031-28] S. E. Han, Massachusetts Institute of Technology (United States); A. Mavrokefalos, Massachusetts Institute of Technology (United States) and The Cypress Institute (Cyprus); M. S. Branham, G. Chen, Massachusetts Institute of Technology (United States)

8031 OU Magnetically responsive photonic nanostructures: making color using magnets (Invited Paper) [8031-29]

L. He, Y. Yin, Univ. of California, Riverside (United States)

\section{SESSION 8 ADVANCED NANOMATERIALS, NANOLITHOGRAPHY AND NANOMANUFACTURING}

8031 OV Functionalized DNA materials for sensing and medical applications (Keynote Paper) [8031-30]

D. L. Woolard, U.S. Army Research Office (United States); J. O. Jensen, U.S. Army Edgewood Chemical Biological Ctr. (United States) 
8031 0X Manufacturing self-assembled coatings of micro- and nano-particles by controlled evaporation of drops and thin films (Invited Paper) [8031-32]

J. Xiao, Columbia Univ. (United States); R. Bhardwaj, The John Hopkins Univ. (United States);

D. Attinger, Columbia Univ. (United States)

8031 OY Graphene optoelectronics based on antidot superlattices (Invited Paper) [8031-33]

S. Strauf, E.-H. Yang, Stevens Institute of Technology (United States)

SESSION 9 MAST-NAVIGATION: JOINT SESSION WITH CONFERENCE 8045

$80310 Z$ Distributed autonomous mapping of indoor environments (Invited Paper) [8031-34]

J. Rogers, M. Paluri, A. Cunningham, H. I. Christensen, Georgia Institute of Technology

(United States); N. Michael, V. Kumar, Univ. of Pennsylvania (United States); J. Ma,

L. Matthies, Jet Propulsion Lab. (United States)

803110 Autonomous navigation with teams of aerial robots (Invited Paper) [8031-35]

N. Michael, V. Kumar, Univ. of Pennsylvania (United States)

803111 Autonomous landing and ingress of micro-air-vehicles in urban environments based on monocular vision (Invited Paper) [8031-36]

R. Brockers, Jet Propulsion Lab. (United States); P. Bouffard, Univ. of California, Berkeley (United States); J. Ma, L. Matthies, Jet Propulsion Lab. (United States); C. Tomlin, Univ. of California, Berkeley (United States)

803112 Velocity estimation using optic flow and radar (Invited Paper) [8031-37]

S. A. Gerardi, J. S. Humbert, Univ. of Maryland, College Park (United States); L. E. Pierce,

K. Sarabandi, Univ. of Michigan (United States)

803113 Compact beam scanning $240 \mathrm{GHz}$ radar for navigation and collision avoidance (Invited Paper) [8031-38]

K. Sarabandi, M. Vahidpour, M. Moallem, J. East, Univ. of Michigan (United States)

SESSION 10 MAST-COMMUNICATION: JOINT SESSION WITH CONFERENCE 8045

803114 New techniques for efficient flexible wireless transceivers in nanometer CMOS (Invited Paper) [8031-39]

M. P. Flynn, D. T. Lin, M. Ghahramani, L. Li, Univ. of Michigan (United States)

803115 Reconfigurable firmware-defined radios synthesized from standard digital logic cells (Invited Paper) [8031-40]

M. Faisal, Y. Park, D. D. Wentzloff, Univ. of Michigan (United States)

803116 Radio signal strength tracking and control for robotic networks (Invited Paper) [8031-41]

P. L. YU, J. N. Twigg, B. M. Sadler, U.S. Army Research Lab. (United States)

803117 Enhanced ad hoc wireless connectivity in complex environment using small radio repeater systems (Invited Paper) [8031-42]

K. Sarabandi, Y. J. Song, J. Oh, Univ. of Michigan (United States) 
803118 Precision navigation and timing enabled by microtechnology: Are we there yet? [8031-43] A. M. Shkel, Defense Advanced Research Projects Agency (United States)

803119 Energy dissipation in micromechanical resonators (Invited Paper) [8031-44]

F. Ayazi, L. Sorenson, R. Tabrizian, Georgia Institute of Technology (United States)

8031 1A Effect of surface chemistry on the quality factors of micromechanical resonators (Invited Paper) [8031-45]

J. A. Henry, Y. Wang, M. A. Hines, Cornell Univ. (United States)

8031 1B Finite element modelling and simulation of thermo-elastical damping of MEMS vibrations (Invited Paper) [8031-46]

S. Kausinis, Kaunas Univ. of Technology (Lithuania); K. Yee, Jet Propulsion Lab. (United States); R. Barauskas, Kaunas Univ. of Technology (Lithuania)

8031 1C Thermal energy loss mechanisms in micro- to nano-scale devices (Invited Paper) [8031-47] A. E. Duwel, J. Lozow, C. J. Fisher, T. Phillips, The Charles Stark Draper Lab. (United States); R. H. Olsson, Sandia National Labs. (United States); M. Weinberg, The Charles Stark Draper Lab. (United States)

\section{SESSION 12 MEMS PERFORMANCE CHALLENGES}

8031 1E An analysis of microsystems development at Sandia National Laboratories (Keynote Paper) [8031-49]

G. V. Herrera, D. R. Myers, Sandia National Labs. (United States)

8031 IF MEMS performance challenges: packaging and shock tests (Invited Paper) [8031-50]

J. Chang, C. Yang, B. Zhang, L. Lin, Univ. of California, Berkeley (United States)

8031 1G Sensors for hydraulic-induced fracturing characterization (Invited Paper) [8031-51] J. Mireles, Jr., Univ. Autónoma de Ciudad Juárez (Mexico); H. Estrada, Ctr. Nacional de Metrología (Mexico); R. C. Ambrosio, Univ. Autónoma de Ciudad Juárez (Mexico)

$8031 \mathrm{lH}$ Tribological challenges in MEMS and their mitigation via vapor phase lubrication (Invited Paper) [8031-52]

M. T. Dugger, Sandia National Labs. (United States)

803111 MEMS and nanostructures: challenges and opportunities. The case of the fight against tuberculosis (Invited Paper) [8031-53]

L. M. Lopez-Marin, Y. D. Contreras-Valeriano, C. Arenas, Univ. Nacional Autónoma de Mexico (Mexico); H. V. Estrada, Ctr. Nacional de Metrología (Mexico); V. M. Castaño, Univ. Nacional Autónoma de Mexico (Mexico) 


\section{Part Two}

\section{SESSION 13 NANOTECHNOLOGIES FOR ENERGY GENERATION AND STORAGE: JOINT SESSION WITH CONFERENCE 8035}

$80311 \mathrm{~J}$ Thermoelectric energy conversion using nanostructured materials (Invited Paper) [8031-54] G. Chen, D. Kraemer, A. Muto, K. McEnaney, H.-P. Feng, Massachusetts Institute of Technology (United States); W.-S. Liu, Q. Zhang, B. Yu, Z. Ren, Boston College (United States)

8031 1K Engineering carbon nanomaterials for future applications: energy and bio-sensor (Invited Paper) [8031-55]

S. Das, I. Lahiri, C. Kang, W. Choi, Florida International Univ. (United States)

8031 1L Developments in MEMS scale printable alkaline and Li-ion technology (Invited Paper) [8031-56]

K. A. Littau, C. L. Cobb, N. Spengler, S. Solberg, M. Weisberg, N. Chang, A. Rodkin, Palo Alto Research Center, Inc. (United States)

$80311 \mathrm{M}$ Further studies in the electrochemical/mechanical strength of printed microbatteries (Invited Paper) [8031-57]

A. Gaikwad, D. Steingart, The City College of New York (United States)

8031 IN 3D architectures are not just for microbatteries anymore (Invited Paper) [8031-58]

J. C. Lytle, Pacific Lutheran Univ. (United States); J. W. Long, C. N. Chervin, M. B. Sassin,

D. R. Rolison, U.S. Naval Research Lab. (United States)

803110 Ultrathin, microscale epitaxial compound semiconductor solar cells (Invited Paper) [8031-59]

J. Yoon, The Univ. of Southern California (United States)

8031 IR Enhanced piezoelectricity and stretchability in energy harvesting devices fabricated from buckled PZT ribbons (Invited Paper) [8031-62]

Y. Qi, Princeton Univ. (United States); P. K. Purohit, Univ. of Pennsylvania (United States);

M. C. McAlpine, Princeton Univ. (United States)

SESSION 14 MICRO- AND NANOTECHNOLOGY FOR HEALTH CARE APPLICATIONS

8031 is Cancer nanotechnology: a new commercialization pipeline for diagnostics, imaging agents, and therapies (Keynote Paper) [8031-63]

K. Ptak, D. Farrell, G. Hinkal, NCl Ctr. for Strategic Scientific Initiatives (United States) and National Institutes of Health (United States); N. J. Panaro, SAIC-Frederick, Inc. (United States) and National Cancer Institute at Frederick (United States); S. Hook, P. Grodzinski, NCl Ctr. for Strategic Scientific Initiatives (United States) and National Institutes of Health (United States)

8031 IT Nanomaterial strategies for immunodetection (Invited Paper) [8031-64]

M. D. Porter, M. C. Granger, L. M. Siperko, The Univ. of Utah (United States); R. J. Lipert, lowa State Univ. (United States) 
$80311 \mathrm{U}$ Nanoparticle targeted therapy against childhood acute lymphoblastic leukemia (Invited Paper) [8031-65]

N. Satake, J. Lee, K. Xiao, J. Luo, S. Sarangi, A. Chang, B. McLaughlin, P. Zhou, E. Kenney,

L. Kraynov, S. Arnott, J. McGee, J. Nolta, K. Lam, Univ. of California, Davis (United States)

8031 1V Optimization of monolithic columns for microfluidic devices (Invited Paper) [8031-66]

J. V. Pagaduan, W. Yang, A. T. Woolley, Brigham Young Univ. Provo (United States)

8031 IW Quantum dots and microfluidic single-molecule detection for screening genetic and epigenetic cancer markers in clinical samples (Invited Paper) [8031-67]

T.-H. Wang, V. Bailey, K. Liu, The Johns Hopkins Univ. (United States)

SESSION 15 MICRO- AND NANOTECHNOLOGY FOR FUTURE HARSH ENVIRONMENT APPLICATIONS

$80311 \mathrm{X}$ Growth of carbon-based nanostructures (Keynote Paper) [8031-68]

W. C. Mitchel, J. J. Boeckl, J. Park, Air Force Research Lab. (United States)

8031 IY Micro- and nano-electronic technologies and their qualification methodology for space applications under harsh environments (Invited Paper) [8031-69]

Y. Chen, NASA Langley Research Ctr. (United States); C. Tudryn Weber, NASA Ames

Research Ctr. (United States); M. Mojarradi, E. Kolawa, Jet Propulsion Lab. (United States)

803121 Graphene field-effect transistors for label-free chemical and biological sensors (Invited Paper) [8031-72]

Y. Ohno, K. Maehashi, K. Matsumoto, Osaka Univ. (Japan)

803122 Effect of energetic electron irradiation on graphene and graphene field-effect transistors (Invited Paper) [8031-73]

I. Childres, M. Foxe, I. Jovanovic, Y. P. Chen, Purdue Univ. (United States)

803123 Xsense: a miniaturised multi-sensor platform for explosives detection (Invited Paper) [8031-74]

M. S. Schmidt, N. Kostesha, F. Bosco, J. K. Olsen, Technical Univ. of Denmark (Denmark);

C. Johnsen, K. A. Nielsen, J. O. Jeppesen, Univ. of Southern Denmark (Denmark); T. S. Alstrøm,

J. Larsen, Technical Univ. of Denmark (Denmark); T. Thundat, Univ. of Alberta (Canada);

M. H. Jakobsen, A. Boisen, Technical Univ. of Denmark (Denmark)

803124 Explosives detection using nanoporous coatings (Invited Paper) [8031-75]

M. P. Pina, I. Pellejero, M. Urbiztondo, J. Sesé, J. Santamaría, Univ. de Zaragoza (Spain)

803125 The photonic nose: a versatile platform for sensing applications (Invited Paper) [8031-76]

L. D. Bonifacio, Opalux, Inc. (Canada); G. A. Ozin, Univ. of Toronto (Canada);

A. C. Arsenault, Opalux, Inc. (Canada) 
803126 Quantum cascade lasers: a game changer for defense and homeland security IR photonics (Keynote Paper) [8031-77]

C. K. N. Patel, Pranalytica, Inc. (United States) and Univ. of California, Los Angeles (United States)

803127 QCL-assisted infrared chemical imaging (Invited Paper) [8031-78]

M. J. Weida, P. R. Buerki, M. Pushkarsky, T. Day, Daylight Solutions Inc. (United States)

803128 Ultrafast bandgap photonics (Invited Paper) [8031-79]

M. K. Rafailov, TRG Inc. (United States)

803129 Vibrational spectroscopy standoff detection of threat chemicals (Invited Paper) [8031-80]

W. Ortiz-Rivera, L. C. Pacheco-Londoño, J. R. Castro-Suarez, H. Felix-Rivera,

S. P. Hernandez-Rivera, Univ. de Puerto Rico Mayagüez (United States)

8031 2B Passive uncooled subMMW focal plane array imager for person-borne explosives detection [8031-113]

J. A. Cox, R. Higashi, F. Nusseibeh, C. Zins, Honeywell ACS Sensors \& Wireless Lab. (United States)

8031 2C Standoff detection of explosives: a challenging approach for optical technologies (Invited Paper) [8031-82]

S. Désilets, Defence Research \& Development Canada, Valcartier (Canada); N. Hô, INO (Canada); P. Mathieu, J. R. Simard, E. Puckrin, J. M. Thériault, H. Lavoie, F. Théberge, Defence Research \& Development Canada, Valcartier (Canada); F. Babin, D. Gay, R. Forest, INO (Canada); J. Maheux, G. Roy, M. Châteauneuf, Defence Research \& Development Canada, Valcartier (Canada)

8031 2D Standoff imaging of chemicals using IR spectroscopy (Invited Paper) [8031-83]

M. E. Morales-Rodríguez, L. R. Senesac, T. Thundat, Oak Ridge National Lab. (United States); M. K. Rafailov, The Reger Group (United States); P. G. Datskos, Oak Ridge National Lab. (United States)

8031 2E Mid-wave/long-wave infrared lasers and their sensing applications (Invited Paper) [8031-84]

K. K. Law, R. Shori, J. K. Miller, S. Sharma, Naval Air Warfare Ctr. Weapons Div. (United States)

POSTER SESSION

$80312 \mathrm{G}$ Integration of CMM software standards for nanopositioning and nanomeasuring machines [8031-86]

E. Sparrer, T. Machleidt, T. Hausotte, E. Manske, K.-H. Franke, Technical Univ. Ilmenau

(Germany) 
$80312 \mathrm{H}$ A novel atomic layer deposition method to fabricate economical and robust large area microchannel plates [8031-87]

A. U. Mane, Q. Peng, Argonne National Lab. (United States); M. J. Wetstein, Argonne National Lab. (United States) and Univ. of Chicago (United States); R. G. Wagner, Argonne National Lab. (United States); H. J. Frisch, Argonne National Lab. (United States) and Univ. of Chicago (United States); O. H. W. Siegmund, Univ. of California, Berkeley (United States); M. J. Minot, Incom Inc. (United States); B. W. Adams, M. C. Chollet, J. W. Elam, Argonne National Lab. (United States)

8031 21 Fabrication of plasmonic nanopore array for biomolecule sensor [8031-88] S. S. Choi, Sun Moon Univ. (Korea, Republic of); M. J. Park, Korea Military Academy (Korea, Republic of); D. S. Kim, N. K. Park, Seoul National Univ. (Korea, Republic of); K. J. Park, National Nanofab Ctr. (Korea, Republic of); T. Yamaguchi, Sun Moon Univ. (Korea, Republic of)

8031 2J Compacted nanoscale sensors by merging ZnO nanorods with interdigitated electrodes [8031-89]

Q. Wang, D. B. Rihtnesberg, A. Bergström, S. Almqvist, A. Z. Z. Zhang, W. Kaplan, J. Y. Andersson, Acreo AB (Sweden); A. Sugunan, X. Yang, M. S. Toprak, Royal Institute of Technology (Sweden)

8031 2K Current injection effects on surface plasmons for a tunable THz source [8031-90]

J. Cleary, Solid State Scientific Corp. (United States); B. Haji-saeed, J. Khoury, C. L. Woods, Air Force Research Lab. (United States); J. Kierstead, Solid State Scientific Corp. (United States)

8031 2L Highly tunable corrugated metal nano-grating laser using current injection [8031-91] J. Khoury, C. L. Woods, Air Force Research Lab. (United States); J. Kierstead, J. Donoghue, Solid State Scientific Corp. (United States)

$80312 \mathrm{~N} \quad$ Zero-bandgap graphene for infrared sensing applications [8031-93] K. W. C. Lai, N. Xi, H. Chen, C. K. M. Fung, L. Chen, Michigan State Univ. (United States)

803120 Scalable fabrication of micro- and nano-particles utilizing the Rayleigh instability in multi-material fibers [8031-94]

S. Shabahang, J. Kaufman, A. F. Abouraddy, CREOL, The College of Optics and Photonics, Univ. of Central Florida (United States)

8031 2P Differential thermal analysis microsystem for explosive detection [8031-95]

J. K. Olsen, A. Greve, Technical Univ. of Denmark (Denmark); L. Senesac, Oak Ridge National Lab. (United States); T. Thundat, Univ. of Alberta (Canada); A. Boisen, Technical Univ. of Denmark (Denmark)

$80312 \mathrm{~T}$ An implementation for the detection and analysis of negative peaks in an applied current signal across a silicon nanopore [8031-99]

J. A. Billo, W. Asghar, S. M. Iqbal, Univ. of Texas at Arlington (United States) and Univ. of Texas Southwestern Medical Ctr. at Dallas (United States)

$80312 \mathrm{U}$ Nanostencil lithography for high-throughput fabrication of infrared plasmonic sensors [8031-100]

S. Aksu, A. A. Yanik, R. Adato, A. Artar, M. Huang, H. Altug, Boston Univ. (United States) 
$80312 \mathrm{~V}$ Cathodoluminescence study of silver and gold lamellar gratings [8031-101]

J. Nath, C. Schwarz, Y. Lin, E. Smith, R. E. Peale, L. Chernyak, Univ. of Central Florida (United States); W. R. Buchwald, Air Force Research Lab. (United States); J. Lee, Univ. of Maryland, College Park (United States)

$80312 X \quad$ Nanosensors: from near field to far field applications [8031-103]

G. M. Herrera, H. Félix, P. M. Fierro, M. Balaguera, L. Pacheco, J. G. Briano, Univ. de Puerto Rico Mayagüez (United States); F. Marquez, Univ. del Turabo-SUAGM (United States); C. Ríos, S. P. Hernández-Rivera, Univ. de Puerto Rico Mayagüez (United States)

$80312 Y \quad$ Plasmonic photonic crystal MEMS emitter for combat ID [8031-104]

I. Puscasu, E. Johnson, A. Taylor, FLIR (ICX) (United States); W. Schaich, Indiana Univ. (United States)

803130 Design of low-cost photonic crystal based three-dimensional invisibility cloak [8031-106] G. Zheng, H. Zhang, Y. Lin, Univ. of North Texas (United States)

803132 Nanowire-based photodetectors: growth and development of chalcogenide nanostructured detectors [8031-108]

M. R. King, S. R. Mclaughlin, D. A. Kahler, A. E. Berghmans, B. P. Wagner, D. J. Knuteson, M. Aziz, N. B. Singh, Northrop Grumman Electronic Systems (United States)

803133 Effect of dielectric layer on the response times of electrostatic MEMS switches [8031-109] S. R. Nelatury, O. Onipede, Pennsylvania State Erie (United States); R. Gray, Pennsylvania State Harrisburg (United States)

803134 Tuneable optical waveguide based on dielectrophoresis and microfluidics [8031-110] A. A. Kayani, A. F. Chrimes, RMIT Univ. (Australia); K. Khoshmanesh, Deakin Univ. (Australia); K. Kalantar-zadeh, A. Mitchell, RMIT Univ. (Australia)

803135 Dielectrophoresis-Raman spectroscopy system for analysing suspended $\mathrm{WO}_{3}$ nanoparticles [8031-111]

A. F. Chrimes, A. Kayani, RMIT Univ. (Australia); K. Khoshmanesh, Deakin Univ. (Australia);

K. Kalantar-zadeh, RMIT Univ. (Australia)

Author Index 


\title{
Conference Committee
}

\author{
Symposium Chair
}

William Jeffrey, HRL Laboratories, LLC (United States)

Symposium Cochair

Kevin P. Meiners, Office of the Secretary of Defense (United States)

Conference Chairs

Thomas George, Zyomed Corporation (United States)

M. Saif Islam, University of California, Davis (United States)

Achyut K. Dutta, Banpil Photonics, Inc. (United States)

Program Committee

Debjyoti Banerjee, Texas A\&M University (United States)

Steve Blair, The University of Utah (United States)

Anja Boisen, Technical University of Denmark (Denmark)

Robert Candler, University of California, Los Angeles (United States)

Scott D. Collins, University of Maine (United States)

Nibir K. Dhar, Defense Advanced Research Projects Agency (United States)

Ernest J. Garcia, Sandia National Laboratory (United States)

Savas Kaya, Ohio University (United States)

Shanalyn A. Kemme, Sandia National Laboratory (United States)

Nobuhiko P. Kobayashi, University of California, Santa Cruz (United States)

Ryan P. Lu, Space and Naval Warfare Systems Command (United States)

Joseph N. Mait, U.S. Army Research Laboratory (United States)

Robert Osiander, The Johns Hopkins University Applied Physics Laboratory (United States)

Nezih Pala, Florida International University (United States)

Jeremy J. Pietron, U.S. Naval Research Laboratory (United States)

Michael K. Rafailov, RICHER International LLC (United States)

Noriko Satake, UC Davis Medical Center (United States)

Andre U. Sokolnikov, Visual Solutions and Applications (United States)

Kyung-Ah Son, Jet Propulsion Laboratory (United States)

Thomas G. Thundat, Oak Ridge National Laboratory (United States)

David V. Wick, Sandia National Laboratory (United States)

Eui-Hyeok Yang, Stevens Institute of Technology (United States)

Karl Y. Yee, Jet Propulsion Laboratory (United States) 
Session Chairs

1 Dip Pen Nanolithography

Ryan P. Lu, Space and Naval Warfare Systems Command (United States)

Debjyoti Banerjee, Texas A\&M University (United States)

2 Advanced Nanoscale Materials Systems

Nobuhiko P. Kobayashi, University of California, Santa Cruz (United States)

3 Micro/Nanotechnology for MM-Wave/THz Security Applications

Robert Osiander, The Johns Hopkins University Applied Physics Laboratory (United States)

$4 \quad \mathrm{THz}$ Characterization of Semiconductor Materials

Andre U. Sokolnikov, Visual Solutions and Applications (United States)

5 MEMS Optical Systems

David V. Wick, Sandia National Laboratory (United States)

Shanalyn A. Kemme, Sandia National Laboratory (United States)

6 Nanophotonics

Steve Blair, The University of Utah (United States)

$7 \quad$ Photon Trapping with 1D Structures and Novel Device Applications

M. Saif Islam, University of California, Davis (United States)

8 Advanced Nanomaterials, Nanolithography and Nanomanufacturing

Eui-Hyeok Yang, Stevens Institute of Technology (United States)

9 MAST-Navigation: Joint Session with Conference 8045

Larry H. Matthies, Jet Propulsion Laboratory (United States)

Joseph N. Mait, U.S. Army Research Laboratory (United States)

10 MAST-Communication: Joint Session with Conference 8045

William Nothwang, U.S. Army Research Laboratory (United States)

Joseph N. Mait, U.S. Army Research Laboratory (United States)

11 Quality Factors for Nano/Micromechanical Resonators

Robert Candler, University of California, Los Angeles (United States)

Thomas George, Zyomed Corporation (United States)

12 MEMS Performance Challenges

Ernest J. Garcia, Sandia National Laboratory (United States) 
13 Nanotechnologies for Energy Generation and Storage: Joint Session with Conference 8035

Jeremy J. Pietron, U.S. Naval Research Laboratory (United States)

Nezih Pala, Florida International University (United States)

14 Micro- and Nanotechnology for Health Care Applications

Scott D. Collins, University of Maine (United States)

Noriko Satake, UC Davis Medical Center (United States)

15 Micro- and Nanotechnology for Future Harsh Environment Applications

Kyung-Ah Son, Jet Propulsion Laboratory (United States)

16 Miniaturized Sensors and Systems

Anja Boisen, Technical University of Denmark (Denmark)

17 Micro-Nanotechnologies for Standoff Detection and Counter Insurgency

Thomas G. Thundat, Oak Ridge National Laboratory (United States)

Michael K. Rafailov, RICHER International LLC (United States) 
Downloaded From: https://www.spiedigitallibrary.org/conference-proceedings-of-spie on 25 Apr 2023

Terms of Use: https://www.spiedigitallibrary.org/terms-of-use 


\section{Introduction}

The 2011 Micro- and Nanotechnology Sensors, Systems, and Applications III conference highlighted recent, cutting-edge developments in a number of diverse topical areas including: Dip-pen Nanolithography, Advanced Nanoscale Materials Systems, Micro/Nanotechnology for $\mathrm{mm}$-Wave/TeraHertz for Security Applications, TeraHertz Characterization of Semiconductor Materials, MEMS Optical Systems, Nanophotonics, Photon Trapping with ID Structures, Advanced Nanomaterials, Nanolithograhy and Nanomanufacturing, Micro Autonomous Sysytems: Navigation and Communication, Quality Factors for Nano/Micromechanical Resonators, MEMS Performance Challenges, Nanotechnologies for Energy Generation and Storage, Micro- and Nanotechnology for Health Care Applications, Micro and Nanotechnology for Future Harsh Environment Applications, Miniaturized Sensors and Systems and Micro-Nanotechnologies for Standoff Detection and Counter-Insurgency.

The sheer breadth of the topics showcased at the conference is indicative of the limitless applications of Micro- and Nanotechnologies. The focus of the conference continues to be on the challenges involved in the transition of these exciting, emerging technologies into practical applications in the Defense, Homeland Security, Space, Medical, and Commercial sectors. Each session is uniquely designed to address three "cornerstones" namely, programmatic investments that set the overall context for the cutting-edge research and development being presented, and the challenges involved in transitioning these exciting concepts to practical, system-level applications. We were, once again, fortunate to showcase advanced micro and nanoscale research being conducted by the Air Force Office of Scientific Research, National Institutes of Health, Department of Energy, Office of Naval Research and the Naval Research Laboratory, Army Research Office and the Army Research Laboratory, NASA, and the Defense Advanced Research Projects Agency.

Thanks to our distinguished contributors, in this proceedings volume you will find papers covering the latest developments in the breathtaking range of topics listed above. We hope you enjoy reading the papers and welcome any suggestions that you may have for future topics of interest to the community.

Thomas George

M. Saif Islam Achyut K. Dutta 
Downloaded From: https://www.spiedigitallibrary.org/conference-proceedings-of-spie on 25 Apr 2023

Terms of Use: https://www.spiedigitallibrary.org/terms-of-use 